\title{
Capacitive MEMS accelerometer wide range modeling using artificial neural network
}

\author{
A. Baharodimehr ${ }^{1}$, A. Abolfazl Suratgar*2, H. Sadeghi ${ }^{3}$ \\ ${ }^{1}$ Department of Electrical Engineering, Arak University, Arak, Iran. \\ 2 Department of Electrical Engineering, Tehran Polytechnic University, Tehran, Iran. \\ *a-suratgar@araku.ac.ir, TEL: +98-861-223-2813, FAX:+98-861-222-5946. \\ ${ }^{3}$ Department of Physics, Arak University, Arak, Iran
}

\begin{abstract}
This paper presents a nonlinear model for a capacitive microelectromechanical accelerometer (MEMA). System parameters of the accelerometer are developed using the effect of cubic term of the folded-flexure spring. To solve this equation, we use the FEA method. The neural network (NN) uses the Levenberg-Marquardt (LM) method for training the system to have a more accurate response. The designed NN can identify and predict the displacement of the movable mass of accelerometer. The simulation results are very promising.
\end{abstract}

Keywords: Accelerometer, MEMS, cubic stiffness, neural network.

\section{RESUMEN}

Este trabajo presenta un modelo no lineal para un acelerómetro microelectromecánico de tipo capacitivo (MEMA). Asimismo, en él se desarrollan parámetros de sistema de el acelerómetro utilizando el efecto del término cúbico del resorte de flexion plegado. Para resolver esta ecuación, usamos el método FEA. La red neuronal (RN) usa el método Levenberg-Marquardt (LM) para entrenar al sistema a fin de que tenga una respuesta más exacta. La RN diseñada puede identificar y predecir el desplazamiento de la masa móvil del acelerómetro. Los resultados de la simulación son muy prometedores.

Palabras clave: Acelerómetro, MEMS,rigidez cúbica, red neuronal.

\section{Introduction}

Nonlinearities in Micro Electro Mechanical System (MEMS) can arise from various sources such as spring and damping mechanisms [1-4], capacitive circuit elements [5], nonlinear coupling between the electrostatic force and displacement on the MEM structure [6], therefore, electrostatic MEMS have nonlinear regions[7].

Several researchers have developed various capacitive MEMS accelerometers [8-11], however, the circuitry design behind the sensing plate of all those accelerometers is based on buffers and demodulators (with low frequency sampling), which is not a suitable response for high accuracy applications. This paper presents a new method to sense the motion of movable plates to have a high accuracy and prevent the device from damage at high accelerations using an artificial neural network [17]. First, a nonlinear dynamic equation with mechanical nonlinearities is obtained. Solving this nonlinear equation can help to determine the displacement of a movable plate with high accuracy, then the applied acceleration at the specific time is sent to the NN to identify and predict the displacement of the movable plate. For training the neural network, the LevenbergMarquardt algorithm is selected.

The accelerometer sensor is a combination of springs, masses and motion sensing and actuation cells as shown in Fig.1 (a). It consists of a variable differential air capacitor whose plates are etched into the suspended polysilicon layer. The moving plate of the capacitor is formed by a large number 
of fingers extending from the beam, a proof mass supported by tethers anchored to the substrate. When responding to an applied acceleration (Fig.1 (b)), the proof mass's inertia causes it to move along a predetermined axis. As the fingers extending from the beam move between the fixed fingers, capacitance change is being sensed and used to measure the amplitude of the force that led to the displacement of the beam.

In Section 2, the analytical model of the MEM accelerometer (MEMA) is obtained. In Section 3, a nonlinear model using the neural network is proposed. Simulation results are given in Section 4. Finally, Section 5 includes the conclusions.

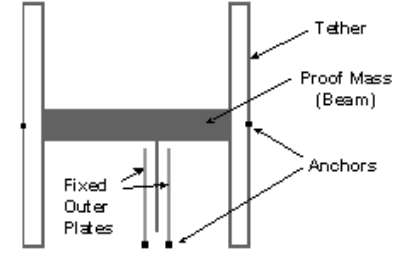

(a)

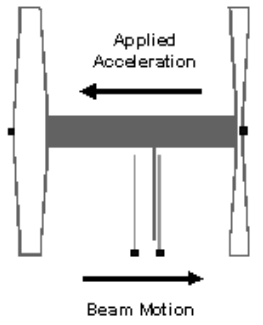

(b)
Figure 1. Schematic diagram of the accelerometer model.

\section{Analytical Modeling of MEMA}

An accelerometer can be modeled as a springmass-damper system in the $\mathrm{x}$-direction. Physicsbased models for the effective spring stiffness of the folded-flexure suspensions, the effective masses of the proof mass and viscous air damping are used in the synthesis tool [12].

Approximations to the nonlinear rod theory provide formulas for the coefficients of linear and cubic stiffening, which enable predictions of spring hardening behavior [1]. Considering the folded- flexure spring and lumped element modeling, the following expression for the spring is obtained:

$$
F_{\text {spring }}=k_{1} \cdot x+k_{3} \cdot x^{3}
$$

Where $k_{1}$ and $k_{3}$ are the linear and cubic stiffness of the folded-flexure spring respectively, they are obtained as follows [1]:

$$
\begin{aligned}
& k_{1}=\frac{12 E I}{L^{3}} \\
& k_{3}=\frac{252 E A}{175 L^{3}}
\end{aligned}
$$

Where ${ }_{E}$ is Young's modulus of polysilicon, $I$ is the moment of inertia of the beam, $L$ is the effective length of the folded-flexure spring and $A$ is the cross section area of the folded-flexure spring.

Therefore, the first linear resonance frequency is given by

$$
\omega_{0}=\sqrt{\frac{k_{1}}{m}}
$$

Where, $m$ is the equivalent lumped mass of the movable plate.

Damping caused by air flow between the rotor and the stator fingers, and at the edge of the proof mass, is the major damping mechanism. For the lateral accelerometer, squeeze-film damping, which occurs when the air gap between two closely placed parallel surface changes, is not critical. The damping coefficient between a single comb finger gap is given as [13]

$$
b=7.2 \mu \cdot l\left(\frac{t}{d}\right)^{3}
$$

Where, $\mu$ is the effective viscosity of air, $t$ is the finger's thickness, $d$ is the finger's gap and $l$ is the finger's length. Table 1 and 2 summarize some properties of the accelerometer's fingers. Table 3 
shows the calculated theoretical lumped parameters for the accelerometer under study.

\begin{tabular}{|c|c|c|c|c|c|}
\hline & $\begin{array}{c}L \\
(\mu \\
\mathrm{m})\end{array}$ & $\begin{array}{c}W_{f} \\
(\mu \mathrm{m})\end{array}$ & $\begin{array}{c}t_{f} \\
(\mu \\
\mathrm{m})\end{array}$ & $\begin{array}{c}d \\
(\mu \\
\mathrm{m})\end{array}$ & $\begin{array}{c}m \\
(\mu \mathrm{g})\end{array}$ \\
\hline $\begin{array}{c}\text { Reference } \\
{[14]}\end{array}$ & $\begin{array}{c}18 \\
0\end{array}$ & 2 & 3 & 1.3 & 0.27 \\
\hline $\begin{array}{c}\text { Reference } \\
{[15]}\end{array}$ & 55 & 3.9 & 5 & 1.5 & 0.57 \\
\hline $\begin{array}{c}\text { Reference } \\
{[13]}\end{array}$ & 50 & 3.9 & 5 & 2 & 0.36 \\
\hline $\begin{array}{c}\text { Reference } \\
{[13]}\end{array}$ & 55 & 3.9 & 5 & 2 & 0.45 \\
\hline $\begin{array}{c}\text { Reference } \\
{[9]}\end{array}$ & 20 & 2 & 2 & 1 & 0.126 \\
\hline
\end{tabular}

Table 1. Specification of some accelerometers.

Where $L_{f}, W_{f}, t_{f}$ are length, width and thickness of the fingers respectively, $f_{r}$ is the resonant frequency, $d$ is the finger gap and $m$ is the mass of the proof mass.

\begin{tabular}{|c|c|}
\hline$E$ (Young's modulus) & $160 \mathrm{GPa}$ \\
\hline$\rho_{m}$ (Density) & $2331 \mathrm{~kg} / \mathrm{m}^{3}$ \\
\hline$v$ (Poisson Ratio) & $\approx 0.2$ \\
\hline
\end{tabular}

Table 2. Properties of the fingers (Material: polysilicon).

\begin{tabular}{|c|c|}
\hline$k_{1}$ & $0.71[\mathrm{~N} / \mathrm{m}]$ \\
\hline$k_{3}$ & $1.136 e 11\left[\mathrm{~N} / \mathrm{m}^{3}\right]$ \\
\hline$m$ & $2.7 e-10[\mathrm{~kg}]$ \\
\hline$b$ & $11.9 e-6[\mathrm{~kg} / \mathrm{s}]$ \\
\hline
\end{tabular}

Table 3. Analytical lumped parameters of the accelerometer.

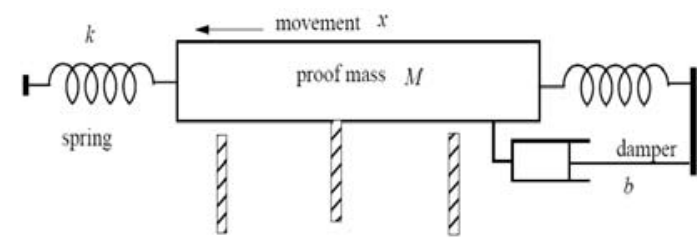

Figure 2. Schematic of a capacitive accelerometer.

In general, considering the equations above and Fig.2, the open-loop nonlinear equation of motion can be written as

$$
m \frac{d^{2} x}{d t^{2}}+b \frac{d x}{d t}+k_{1} x+k_{3} x^{3}=m a_{e x t}
$$

Where, $a_{\text {ext }}$ is the applied acceleration from environment, $x$ is the center displacement of proof mass. The response of this equation is calculated using the Runge-Kutta method and shown in Fig.4.

\section{Nonlinear Model of MEMA Using an Artificial Neural Network}

Figure 1 shows the vertical section at constant $12 w t . \%$ (5.3at.\%) $\mathrm{Zn}$ of the Al-Zn-Mg phase diagram [13], where the vertical lines indicate the $\mathrm{Mg}$ content added to the Al-12wt. \%Zn master alloy. The microstructures are constituted mainly by columnar dendrites of $\alpha$-Al with small $\tau$ $\left(\mathrm{Al}_{2} \mathrm{Mg}_{3} \mathrm{Zn}_{3}\right)$ precipitates and eutectic $(\alpha+\tau)$ in interdendritic regions, as is shown in Figure 2 and in agreement with the work of Alvarez et al. [14].

The ANN's inputs are applied accelerations and time of actuating of the specific acceleration applied to the device. The output of the ANN is the displacement of the movable plate; here, a three layer feed-forward network is created (Fig.3). The first layer has 22 linear transfer functions, the second layer has 10 hyperbolic tangent sigmoid transfer functions and the last layer has one linear transfer function. 


\section{Capacitive MEMS accelerometer wide range modeling using artificial neural network, A. Baharodimehr et, al, 185-192}

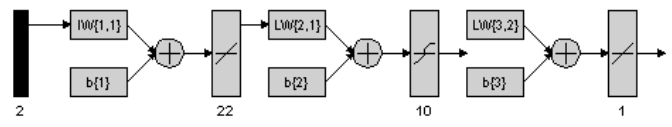

Figure 3. Neural Network Design.

For training the network, we suggest the Levenberg-Marquardt (LM) algorithm. In the following, the Levenberg-Marquart method is reviewed [16]. In the EBP algorithm, the performance index $F(w)$ to be minimized is defined as the sum of squared errors between the target outputs and the network's simulated outputs, namely:

$$
F(w)=e^{T} e
$$

Where, $w=\left[w_{1}, w_{2}, \ldots, w_{N}\right]$ consists of all weights of the network, $e$ is the error vector comprising the error for all the training examples.

When training with the LM method, the increment of weights $\Delta w$ can be obtained as follows:

$$
\Delta w=\left[J^{T} J+\mu I\right]^{-1} J^{T} e
$$

Where, $\mathrm{J}$ is the Jacobian matrix, $\mu$ is the learning rate which is to be updated using the $\beta$ depending on the outcome. In particular, $\mu$ is multiplied by the decay rate $\beta \quad(0<\beta<1)$ whenever $F(w)$ decreases, whereas $\mu$ is divided by $\beta$ whenever $F(w)$ increases in a new step.

The standard LM training process can be illustrated in the following pseudo-codes:

1. Initialize the weights and parameter $\mu$ ( $\mu=.01$ is appropriate).

2. Compute the sum of the squared errors over all inputs $F(w)$.

3. Solve (8) to obtain the increment of weights $\Delta w$ 4. Recompute the sum of squared errors $F(w)$
Using $w+\Delta w$ as the trial $w$, and judge IF trial in step 2 THEN

$$
\begin{gathered}
w=w+\Delta w \\
\mu=\mu \cdot \beta(\beta=.1)
\end{gathered}
$$

Go back to step 2

ELSE

$$
\mu=\mu / \beta
$$

go back to step 4

END

Considering the performance index is $F(w)=e^{T} e$ using the Newton method we have:

$W_{K+1}=W_{K}-A_{K}^{-1} \cdot g_{K}$

$$
\begin{aligned}
& A_{k}=\left.\nabla^{2} F(w)\right|_{w=w_{k}} \\
& g_{k}=\left.\nabla F(w)\right|_{w=w_{k}} \\
& {[\nabla F(w)]_{j}=\frac{\partial F(w)}{\partial w_{j}}} \\
& =2 \sum_{i=1}^{N} e_{i}(w) \cdot \frac{\partial e_{i}(w)}{\partial w j}
\end{aligned}
$$

The gradient can be written as

$$
\nabla F(x)=2 J^{T} e(w)
$$

Where,

$$
J(w)=\left[\begin{array}{l}
\frac{\partial e_{11}}{\partial w_{1}} \frac{\partial e_{11}}{\partial w_{2}} \cdots \cdots \cdot \frac{\partial e_{11}}{\partial w_{N}} \\
\frac{\partial e_{21}}{\partial w_{1}} \frac{\partial e_{21}}{\partial w_{2}} \cdots \cdots \frac{\partial e_{21}}{\partial w_{N}} \\
\vdots \\
\frac{\partial e_{K P}}{\partial w_{1}} \frac{\partial e_{K P}}{\partial w_{2}} \cdots \cdots \frac{\partial e_{K P}}{\partial w_{N}}
\end{array}\right]
$$

$J(w)$ is called the Jacobian matrix. 
Next, we want to find the Hessian matrix. The $k$, j elements of the Hessian matrix yields as

$$
\begin{aligned}
{\left[\nabla^{2} F(w)\right]_{k, j} } & =\frac{\partial^{2} F(w)}{\partial w_{k} \partial w_{j}} \\
= & 2 \sum_{i=1}^{N}\left\{\frac{\partial e_{i}(w)}{\partial w_{k}} \frac{\partial e_{i}(w)}{\partial w_{j}}\right. \\
& \left.+e_{i}(w) \cdot \frac{\partial^{2} e_{i}(w)}{\partial w_{k} \partial w_{j}}\right\}
\end{aligned}
$$

The Hessian matrix can then be expressed as follows:

$\nabla^{2} F(W)=2 J^{T}(W) \cdot J(W)+S(W)$

Where,

$S(w)=\sum_{i=1}^{N} e_{i}(w) \cdot \nabla^{2} e_{i}(w)$

If we assume that $S(w)$ is small, we can approximate the Hessian matrix as

$\nabla^{2} F(w) \cong 2 J^{T}(w) J(w)$

Using (12) and (4), we obtain the Gauss-Newton method:

$W_{k+1}=$

$W_{k}-\left[2 J^{T}\left(w_{k}\right) \cdot J\left(w_{k}\right)\right]^{-1} 2 J^{T}\left(w_{k}\right) e\left(w_{k}\right)$

$\cong W_{k}-\left[J^{T}\left(w_{k}\right) \cdot J\left(w_{k}\right)\right]^{-1} J^{T}\left(w_{k}\right) e\left(w_{k}\right)$

The advantage of the Gauss-Newton method is that it does not require calculation of second derivatives.

There is a problem with the Gauss-Newton method: the matrix $\mathrm{H}=\mathrm{JTJ}$ may not be invertible. This can be overcome by using the following modification:
The Hessian matrix can be written as

$G=H+\mu I$

Let us suppose that the eigenvalues and eigenvectors of $H$ are $\{\lambda 1, \lambda 2, \ldots \ldots ., \lambda n\}$ and $\{z 1, z 2, \ldots \ldots . ., z n\}$.Then

$$
\begin{aligned}
G z_{i}= & {[H+\mu I] z_{i} } \\
& =H z_{i}+\mu z_{i} \\
& =\lambda_{i} z_{i}+\mu z_{i} \\
& =\left(\lambda_{i}+\mu\right) z_{i}
\end{aligned}
$$

Therefore, the eigenvectors of $G$ are the same as the eigenvectors of $H$, and the eigenvalues of $G$ are $(\lambda i+\mu)$. Matrix $G$ is positive definite by increasing $\mu$ until $(\lambda i+\mu)>0$ for all $i$, therefore, the matrix will be invertible.

This leads to the Levenberg-Marquardt algorithm:

$w_{K+1}=w_{K}-\left[J^{T}\left(w_{K}\right) J\left(w_{k}\right)+\mu I\right]^{-1} J^{T}\left(w_{K}\right) e\left(w_{K}\right)$

$\Delta w_{K}=\left[J^{T}\left(w_{K}\right) J\left(w_{K}\right)+\mu I\right]^{-1} J^{T}\left(w_{K}\right) e\left(w_{K}\right)$

As it is known, the learning parameter $\mu$ illustrates the steps of the actual output movement to the desired output. In the standard LM method, $\mu$ is a constant number.

In this paper, the input matrix for training the NN has $2 * 6000$ dimensions and the output matrix has $1 * 6000$ dimensions. After training the $\mathrm{NN}$, for testing it, a $2 * 120$ matrix is given to the input of the $\mathrm{NN}$ and the results are evaluated.

\section{Simulation Results}

By using Eq. (6), the displacement response of a movable plate with $10 \mathrm{~g}\left(\approx 100 \mathrm{~m} / \mathrm{s}^{2}\right)$ acceleration is calculated and shown in Fig.4. 


\section{Capacitive MEMS accelerometer wide range modeling using artificial neural network, A. Baharodimehr et, al, 185-192}

To validate Fig.4, we use the FEA analysis for modeling this accelerometer. The results are shown in Fig.5.

With regard to the cubic stiffness of the spring, the results are more accurate (Fig.6), therefore, when the ANN is trained, its output can be fit with the diagram that regards the cubic stiffness (Fig.7).

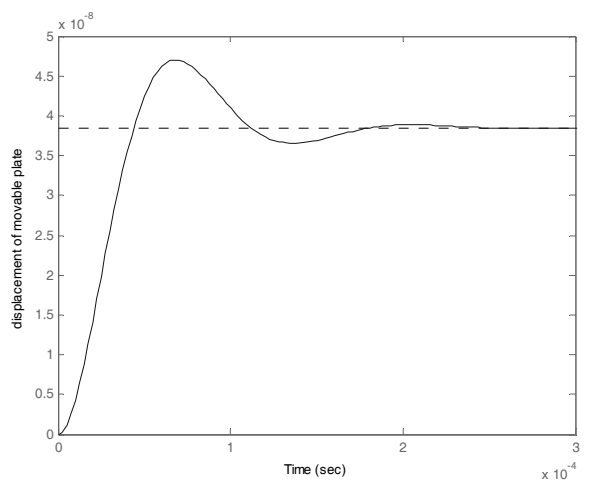

Figure 4.Calculated displacement response.

One of the advantages of this method is that we can predict the damage to the fingers of the device because of the high acceleration, so an electrical feedback to the movable plate can prevent the damage.

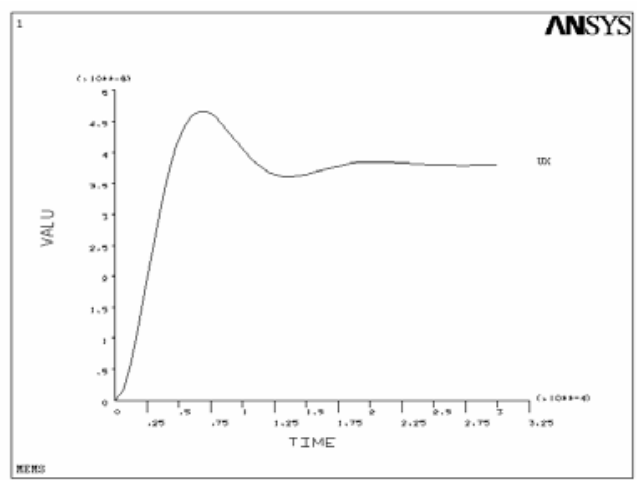

Figure 5. Displacement of the movable plate with the FEA method.

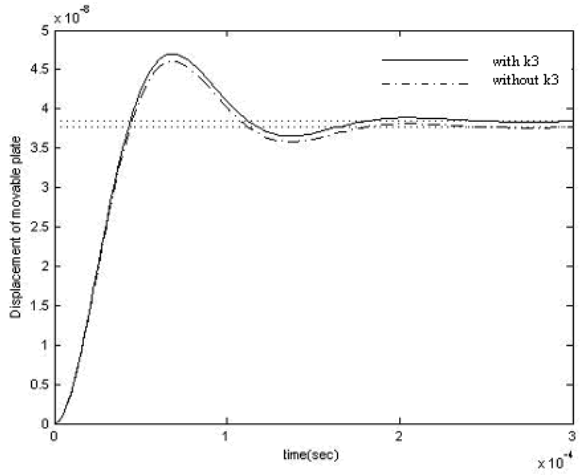

Figure 6. Comparison between the calculated displacement response with $\mathrm{k} 3$ and without $\mathrm{k} 3$.

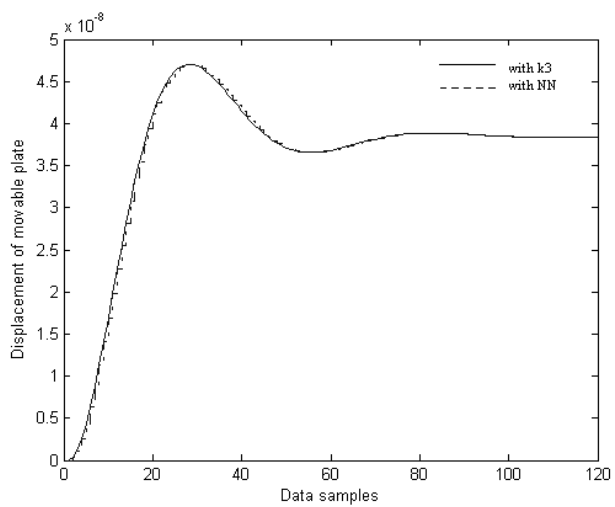

Figure 7. Output of the NN with a test data with $10 \mathrm{~g}$ acceleration.

\section{Conclusions}

This paper presented a nonlinear model for a capacitive MEMA. System parameters of the accelerometer were developed using the effect of cubic term of the folded-flexure spring. To solve this equation, the FEA method was used. The artificial neural network (ANN) used the Levenberg-Marquardt (LM) method for training the system to have a more accurate response. The designed ANN identified and predicted the displacement of the movable mass of the accelerometer. The simulation results were very promising. 
The neural network is a good method to predict the response with very high accuracy. The response of the device using cubic stiffness is more accurate, therefore, the ANN acts better than the linear model.

\section{References}

[1] W. L. Davis, "Mechanical Analysis and Design of Vibratory Micromachined", Ph.D. thesis, University of California, Brekeley, 2001.

[2] P. Malatkar, "Nonlinear Vibrations of Cantilever Beams and Plates", Ph.D thesis, Faculty of the Virginia Polytechnic Institue and State University,2003.

[3] Q. Jing, "Modeling and Simulation for Design of Suspended MEMS", Ph.D. thesis, Carnegie Mellon University, 2003.

[4] S.V. Iyer, "Modeling and Simulation of Non-idealities in a Z-axis CMOS-MEMS Gyroscope", Ph.D. thesis, Carnegie Mellon University, 2003.

[5] J. Bienstman, R. Puers, and J. Vandewalle, "Periodic and chaotic behavior of the autonomous impact resonator",IEEE Proc. MEMS'98, pp.562-567,1998.

[6] A.F. El-Bassiouny and M. Eissa, "Dynamics of a singledegree-of-freedom structure with quadratic, cubic and quartic nonlinearities to a harmonic resonance",APPL. Math. Comput.,vol. 139,pp.1-21, 2003.

[7] S.K. De and N.R. Aluru, "Complex Nonlinear Oscillation in Electrostatically Actuated Microstructures",Journal of Microelectromechanical Systems, Vol.15, NO. 2, 2006.

[8] S. Rekuc, "Optimization for a Robust MEMS Accelerometer Spring", Final project, Georgia institute of technology, The G. W. Woodroof School of Mechanical Engineering, 2003.

[9] D. P. Beach, "Design of an Open Loop Capacitive Accelerometer", Mini-thesis, Indiana State University, The College of Technology,2004.
[10] T. Veijola, H. Kuisma and J. Lahdenpera, "Compact Large-Displacement Model for a Capacitive Accelerometer", MSM'99, pp. 218-221, 1999.

[11] V. Gupta, "Approaches to Synthesis of a CMOS Accelerometer", M.Sc. Thesis, Carnegie Mellon University, 2000.

[12] G. K. Fedder, "Simulation of Micro-electromechanical Systems", Ph.D thesis, University of California, Berkeley, 1994.1.

[13] G. Zhang, "Design and Simulation of a CMOS-MEMS Accelerometer", M.Sc. thesis, Carnegie Mellon University,1998.

[14] A. K. Sharma and A. Teverovksy, "Evaluation of Thermo-Mechanical Stability of COTS Dual-Axis MEMS Accelerometers for Space Applications", Component Technology and Radiation Effects (Code 562), 2000.

[15] H. Luo, "Integrated Multiple Device CMOS-MEMS IMU Systems and RF MEMS Applications", Ph.D thesis, Carnegie Mellon University,2002

[16] A. A. Suratgar and M. B. Tavakoli and A. Hossenabadi, "Modified Levenberg-Marquardt neural network training", Enformatika , 2005.

[17] A. A. Suratgar and A. R. Bahadorimehr and $H$. Sadeghi and M. Dousti, "Nonlinear Modeling of a Capacitive MEMS Accelerometer Using Neural Network", in proceedings of EMERGING MECHANICAL TECHNOLOGY Macro to Nano conference, Delhi, India, 2007. 


\section{Authors Biography}

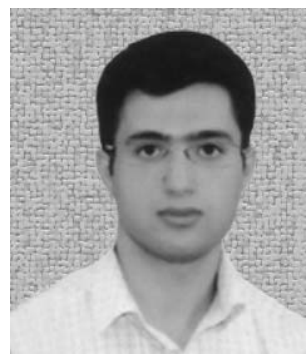

\section{Alireza R. BAHADORIMEHR}

He was born in Iran in 1982. He received his BS and MS degrees in electronics engineering from Arak Azad University in 2004 and 2006, respectively. He is now a PhD candidate at UKM University. His main interests are in the field of Bio-MEMS and Lab-On-Chip using microfluidic devices.

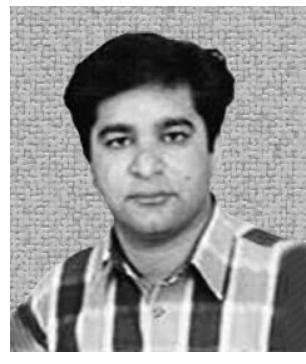

\section{Hossein SADEGHI}

Dr. Sadeghi received his B. S., M. S. and Ph. D. degrees in theoretical physics from The University of Tehran, Tehran, Iran, in 1997, 2000 and 2005, respectively. He is currently an assistant professor in the Physics Department of The University of Arak. His research interest areas are low energy properties of hadrons by non-perturbative solutions of the strong interactions theory, Quantum Chromo Dynamics (QCD). He has published more than 10 papers in theoretical nuclear and solid states physics and 50 papers in conferences.

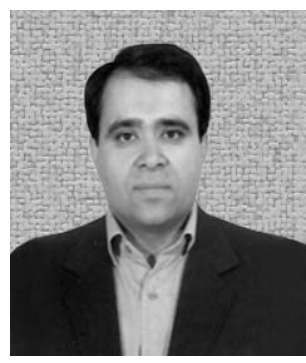

\section{Amir ABOLFAZL-SURATGAR}

Dr. Suratgar was born in Arak, Iran, in 1974. He received his B. S. degree in electrical engineering with honors from Isfahan University of Technology, Isfahan, Iran, in 1996 and the M. S. and Ph. D. degrees in control engineering from Amirkabir University of Technology (Tehran Polytechnic), Tehran, Iran, in 1999 and 2002, respectively. He is an assistant professor of electrical engineering at the University of Arak, Arak, Iran. Dr. Suratgar received the Outstanding Research Award from the Faculty of Engineering of The University of Arak in 2006 and 2005. Also, he received the Outstanding Education Award from the Faculty of Engineering of The University of Arak in 2009. Currently, he collaborates with Amirkabir University of Technology (Tehran Polytechnic), Tehran, Iran. His research interest areas are among system stability, system modeling and micro electro mechanical systems. He has published more than 50 papers in scientific journals and conferences. 